\title{
Neutrinos and the Highest Energy Cosmic Rays
}

\author{
A. Letessier-Selvon ${ }^{\mathrm{a} *}$ \\ ${ }^{a}$ Laboratoire de Physique Nucléaire et des Hautes Énergies, \\ IN2P3-CNRS, Universités Paris 6 \& 7, \\ 4 place Jussieu, Tour 33 RdC, 75252 Paris Cedex 05 France
}

\begin{abstract}
Observation of Ultra High Energy Cosmic Rays (UHECR) -whose energy exceeds $10^{20} \mathrm{eV}$ - is still a puzzle for modern astrophysics. The transfer of more than 16 Joules to a microscopic particle can hardly be achieved, even in the most powerful cosmic accelerators such as AGN's, GRB's or FR-II radio galaxy lobes. Potential sources must also lie within $100 \mathrm{Mpc}$ of the Earth as the interaction length of protons, nuclei or photons is less than $10 \mathrm{Mpc}$. However no visible counterpart of those sources has been observed. Calling upon new physics such as Topological Defect interactions or Super Massive Relic Particle decays is therefore very tempting, but such objects are yet to be proven to exist. Due to the very low flux of UHECR only very large dedicated experiments, such as the Auger observatories, will allow to shed some light on the origin of those cosmic rays. In this quest neutrinos, if they can be detected, are an invaluable messengers of the nature of the sources.
\end{abstract}

\section{INTRODUCTION}

The cosmic ray spectrum is now proved [1],2] to extend beyond $10^{20} \mathrm{eV}$. To be observed on Earth with such energies, particles must be produced or accelerated in the Universe with energy near or above $10^{21} \mathrm{eV}$. Conventional acceleration mechanisms in astrophysical objects can only reach this requirement by stretching to the limit their available parameter space, making such scenarios unlikely to explain the origin of UHECR. Alternative hypothese involving collapse of Topological Defects (TD) or decay of Super Massive Relic Particles (SMRP) are well suited to produce particles above $10^{20} \mathrm{eV}$ but need a proof of existence.

Transport, from the source to Earth, is also an issue. At those extreme energies the Cosmic Microwave Background Radiation makes the Universe essentially opaque to protons, nuclei and photons which suffer energy losses from pion photo production, photo-disintegration or pair production. These processes led Greisen, Zatsepin and Kuzmin[3] to predict a spectral cutoff in the cosmic ray spectrum around $5 \times 10^{19} \mathrm{eV}$, the GZK cutoff. The available data although still very scarce do not support the existence of such

\footnotetext{
*e-mail : Antoine.Letessier-Selvon@in2p3.fr
}

a cutoff. Therefore the sources are either close by and locally over dense for the cutoff not to show, or new physics prevents the UHECR from the expected energy losses against the CMB photons.

The following will briefly develop the arguments mentioned in this introduction. Interested readers should consult the numerous reviews de-

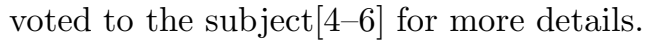

\section{OBSERVATIONS}

The differential spectrum of cosmic ray flux [7] as a function of energy is shown on Figure 1 . Integrated fluxes above three energy values are indicated: 1 particle $/ \mathrm{m}^{2}$-second above $1 \mathrm{TeV}, 1$ particle $/ \mathrm{m}^{2}$-year above $10 \mathrm{PeV}, 1$ particle $/ \mathrm{km}^{2}$ year above $10 \mathrm{EeV}$. The energy spectrum is surprisingly regular in shape. From the $\mathrm{GeV}$ energies to the GZK cutoff, it can be represented simply by three power-law segments interrupted by two breaks, the so-called "knee" and "ankle".

Figure 2 is a zoom on the highest energy part of the total spectrum where only the latest AGASA data[2] is displayed. On this figure, the energy spectrum is multiplied by $E^{3}$ so that the part below the $\mathrm{EeV}$ energies becomes flat. Comparing the data points and the dashed line one has a 


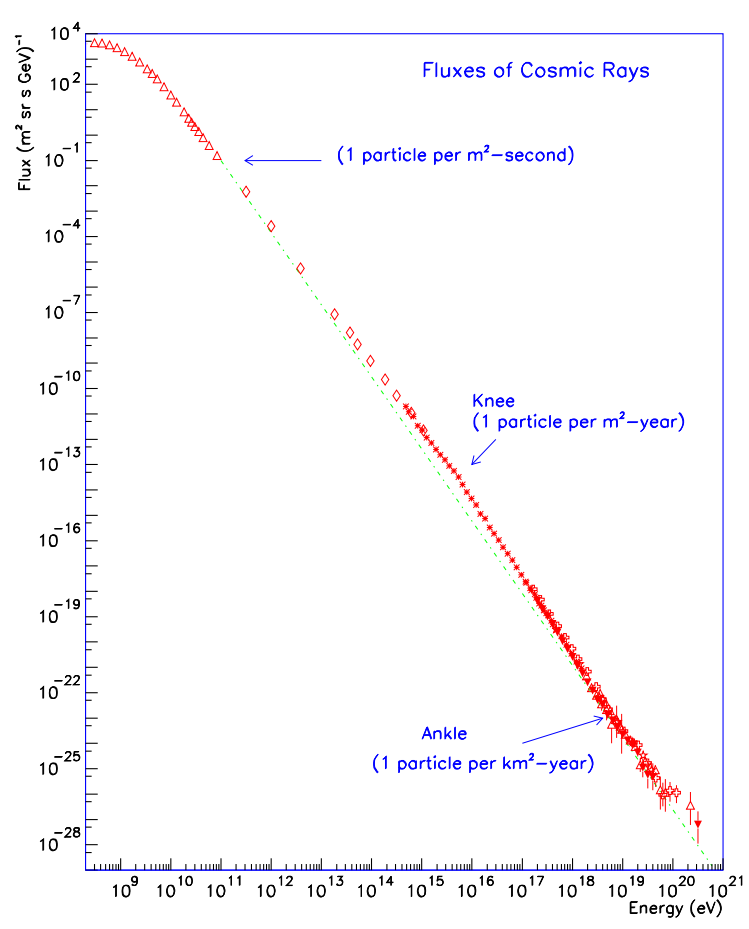

Figure 1. The cosmic rays spectrum[7]

clear view of what can be expected from a cosmological (uniform) distribution of conventional sources and what is observed.

The cutoff, that would be expected if the sources were cosmologically and uniformly distributed and if the observed cosmic rays had no exotic propagation or interaction properties, is not present in the observed data.

In the search for potential sources, one looks for correlations of the UHECR arrival directions with the distribution of matter within a few tens of Mpc. Such an analysis was done by the AGASA experiment for the highest energy range [8]. No convincing deviation from isotropy was found.

If the sources of UHECR are nearby astrophysical objects and if, as expected, they are in small numbers, a selection of the events with the largest magnetic rigidity would combine into multiplets (cluster of events whose error boxes overlap).

Figure 3 shows the subsample of events in the AGASA catalog with energies in excess of $100 \mathrm{EeV}$ (squares) and in the range 40-100 EeV

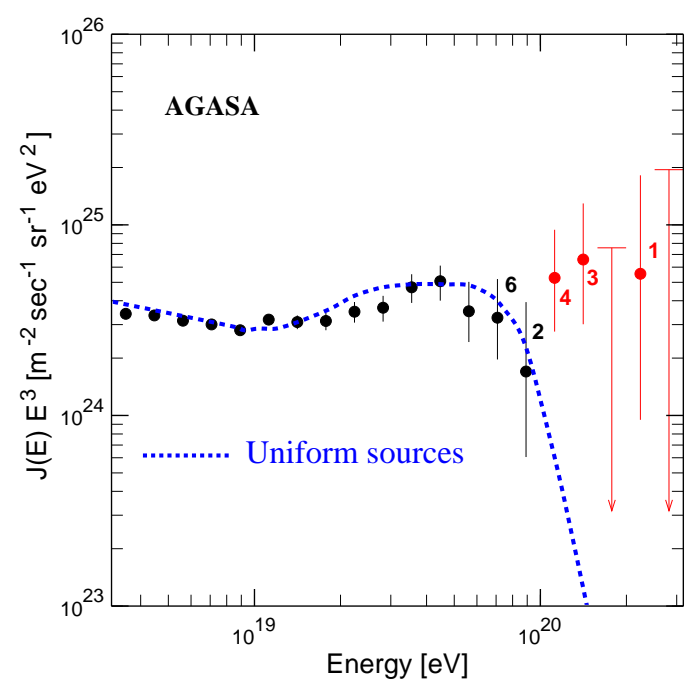

Figure 2. Highest energy region of the cosmic ray spectrum as observed by the AGASA[2] detector.

(circles). One can see that there are three doublets and one triplet. The chance probability of having as many multiplets as observed with a uniform distribution is estimated to be less than $1 \%$ [8].

The non uniform sky coverage -all present detectors are in the northern hemisphere- and the small statistics available make anisotropy studies difficult. The Auger observatories are designed with full sky coverage and large detection areas to overcome these difficulties.

\section{TRANSPORT AND PRODUCTION}

Today's understanding of the phenomena responsible for the production of UHECR is still limited. One distinguishes two classes of processes: the "Top-Down" and "Bottom-Up" scenarios. In Top-Down scenarios, the cosmic ray is a decay products of a super-massive particle. Such particles with masses exceeding $10^{21} \mathrm{eV}$ are either meta-stable relics of some primordial field or a GUT gauge boson produced by the radiation or collapse of topological defects. In the Bottom-Up scenarios, the energy is transferred to a charged particle at rest through its electromag- 


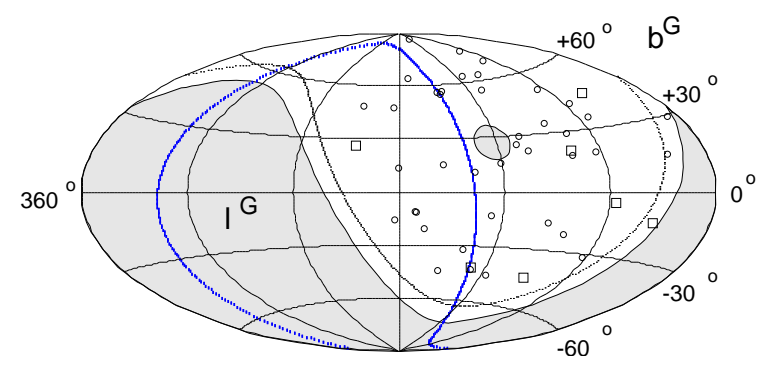

Figure 3. Arrival directions (galactic coordinates) of cosmic rays with $E>40 \mathrm{EeV}$, AGASA. [8]

netic interactions. This classical approach does not require new physics.

At energies above $10 \mathrm{EeV}$ and except for neutrinos, the Universe is not transparent to ordinary stable particles on scales larger than about 100 Mpc. Regardless of their nature, cosmic rays lose energy in their interaction with the various photon backgrounds, dominantly the copious Cosmic Microwave Background (CMB) but also InfraRed and Radio. The absence of prominent visible astrophysical objects in the direction of the observed highest energy cosmic rays together with this distance limitation adds severe constraints on the "classical" Bottom-Up picture.

\subsection{GZK cutoff}

The energy at which the Greisen-ZatsepinKuzmin (GZK) cutoff takes place is given by the threshold for pion photo-production in the proton CMB-photon collisions. For an average CMB photon $\left(10^{-3} \mathrm{eV}\right)$, one obtains $E_{t h}=7 \times 10^{19} \mathrm{eV}$. The interaction length can be estimated from the pion photo-production cross section and the $\mathrm{CMB}$ density : $L=(\sigma \rho)^{-1} \simeq 6 \mathrm{Mpc}$.

A recent Monte Carlo [9] calculation, including red shift, pair production and pion photoproduction losses, is shown on Figure 4 . The loss length $x_{\text {loss }}$ is defined as $x_{\text {loss }}=\frac{E}{d E / d x}$. Above $100 \mathrm{EeV}$ photo-production processes are dominant and the loss length falls below $13 \mathrm{Mpc}$.

For nuclei, the situation is usually worse. They photo-disintegrate in the CMB and infrared radiations losing on average 3 to 4 nucleons per $\mathrm{Mpc}$ when their energy exceeds $2 \times 10^{19} \mathrm{eV}$ to $2 \times 10^{20} \mathrm{eV}$ depending on the IR background density value.

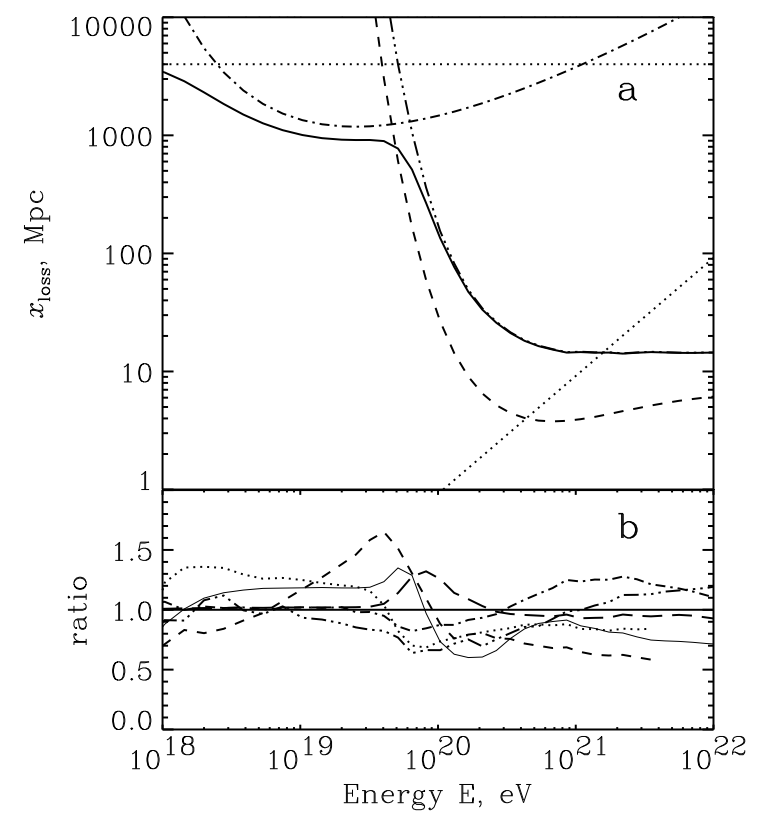

Figure 4. Top : Loss length of a proton from [9]. Bottom : ratios with other calculations.

Top-Down production mechanisms predict that, at the source, photons and neutrinos dominate over ordinary hadrons by about a factor four to ten [5.11]. An observed dominance of gammas or neutrinos in the supra-GZK range would then be an inescapable signature of a super-heavy particle decay or TD interaction. High energy photons traveling through the Universe produce $e^{+} e^{-}$ pairs when colliding with the Infra-Red/Optical (IR/O), CMB or Universal Radio Background (URB) photons. As can be seen on Figure 5 the attenuation length gets below $10 \mathrm{Mpc}$ for photon energies between $3 \times 10^{13} \mathrm{eV}$ and $10^{20} \mathrm{eV}$. In this energy range the Universe is opaque to photons on cosmological scales.

Once the photon has converted, the $e^{+} e^{-}$pair will in turn produce photons mostly via Inverse Compton Scattering (ICS). Those two dominant processes are responsible for the production of electromagnetic (EM) cascades. On Figure 5 one sees that, at the pair production threshold on the CMB photons $\left(2 \times 10^{14} \mathrm{eV}\right)$, conversion occurs on distances of about $10 \mathrm{kpc}$ (a thousand times smaller than for protons at GZK energies) while 


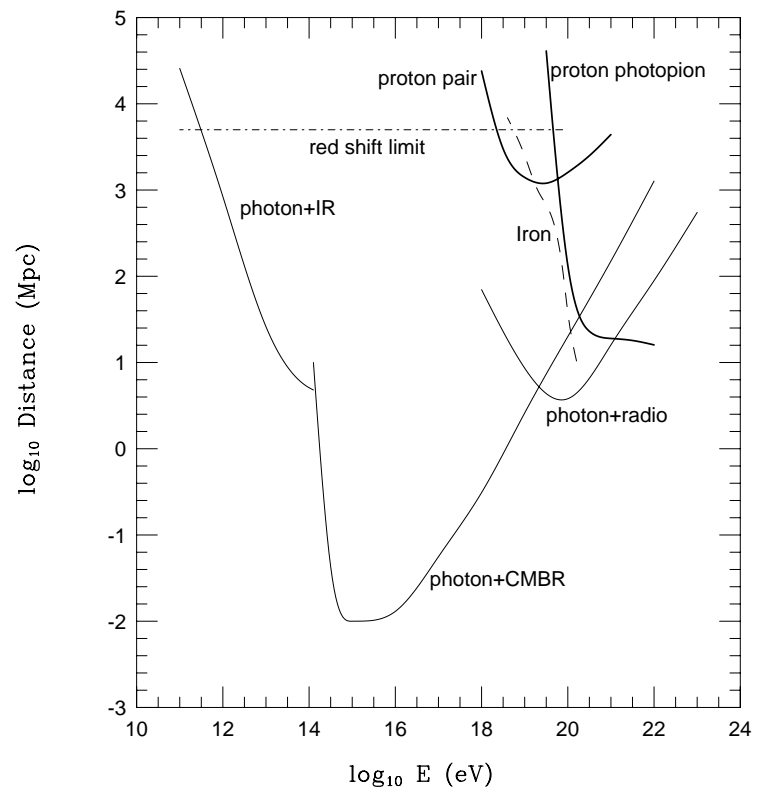

Figure 5. Attenuation length of photons, protons and iron. Double pair production (not shown) limits the photon attenuation length to about $100 \mathrm{Mpc}$ above $10^{22} \mathrm{eV}$.10

subsequent ICS of electrons on the CMB in the Thomson regime will occur on even smaller scales (1 kpc).

As a consequence, most photons of ultra high energy will produce, through successive collisions on the various photon backgrounds (URB, CMB, IR/O), lower and lower energy cascades and pile up in the form of a diffuse photon background below $10^{12} \mathrm{eV}$ with a typical power law spectrum of index $\alpha=1.5$. This is a very important fact as measurements of the diffuse gamma ray background in the $10^{7}-10^{11} \mathrm{eV}$ range done for example by EGRET 12 will impose limits on the photon production fluxes of Top-Down mechanisms and consequently on the abundance of topological defects or relic super-heavy particles.

Neutrinos are the only known particles that can travel through space unaffected even on large distances, carrying intact the properties of the source to the observer. They may prove to be an unambiguous signature of the new physics underlying the production mechanisms.

\subsection{Bottom-Up acceleration}

In the conventional acceleration scenarios one distinguishes two types of mechanisms :

- Direct acceleration by very high electric fields in or near very compact objects. This does not naturally provide a power-law spectrum.

- Diffusive shock acceleration in all systems where shock waves are present. This statistical acceleration, known as the Fermi mechanism, naturally provides a power-law spectrum.

Hillas has shown 13 that irrespective of the details of the acceleration mechanisms, the maximum energy of a particle of charge $Z e$ within a given site of size $R$ is:

$E_{\max } \approx \beta Z\left(\frac{B}{1 \mu \mathrm{G}}\right)\left(\frac{R}{1 \mathrm{kpc}}\right) 10^{18} \mathrm{eV}$

where $B$ is the magnetic field inside the acceleration volume and $\beta$ the velocity of the shock wave or the efficiency of the acceleration mechanism. This condition is nicely represented by the Hillas diagram shown in Figure 6. Inspecting this diagram one sees that only a few astrophysical sources satisfy the necessary condition given by Eq. (1). Let us briefly review them :

Pulsars : From a dimensional analysis, one obtains up to $10^{20}$ volts for the potential drop in a rotating magnetic pulsar. However the high radiation density in the vicinity of the pulsar will produce $e^{+} e^{-}$pairs which reduce the potential drop down to values of about $10^{13} \mathrm{eV}$. A different mechanisms involving Fe nuclei acceleration by relativistic MHD winds has been put forward [14]. But details of the effectiveness of this mechanism still need to be demonstrated.

AGN cores and jets : Blast waves in AGN jets could in principle lead to a maximum energy of a few tens of EeV [15] and similarly for AGN cores. However those maxima are unlikely to be achieved under realistic conditions due to the interaction of the accelerated protons with very high radiation fields in and around the central engine of an AGN. 


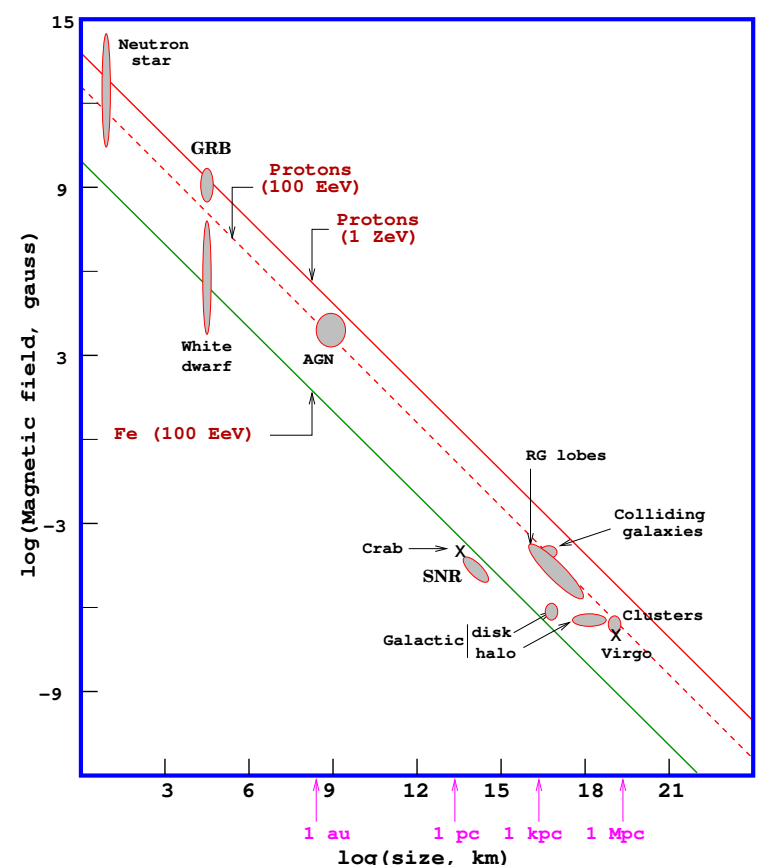

Figure 6. Size and magnetic field strength of possible acceleration sites. Objects below the diagonal lines cannot accelerate the corresponding elements above $10^{20} \mathrm{eV}$ or $10^{21} \mathrm{eV}$.

FR-II radio galaxies : Radio-loud quasars are characterized by a very powerful central engine ejecting matter along thin extended jets. At the ends of those jets, the so-called hot spots, the relativistic shock wave is believed to be able to accelerate particles up to $\mathrm{ZeV}$ energies. FRII galaxies seem the best potential astrophysical source of UHECR 16]. Unfortunately, no nearby (less than $100 \mathrm{Mpc}$ ) object of this type is visible in the direction of the observed highest energy events.

Gamma Ray Burst : Gamma ray bursters (GRB) are intense sources of gamma rays. The most favored GRB emission model is the "expanding fireball model" where one assumes that a large fireball, as it expands, becomes optically thin hence emitting a sudden burst of gamma rays. The observation of afterglows allowed measurement of the red shift of the GRBs hence confirming their cosmological origin. GRB can be shown to accelerate protons up to $10^{20} \mathrm{eV}[17$.
However in such a framework the UHECR spectrum should clearly show the GZK cut-off.

\subsection{Top-Down production}

One way to overcome problems related to the acceleration of UHECR and the invisibility of their sources is to introduce a new unstable or meta-stable super-massive $X$-particle. The decay of this $X$-particle produces, among other things, quarks and leptons, resulting in a large cascade of energetic photons, neutrinos and light leptons with a small fraction of protons and neutrons, part of which becomes the UHECR. For this scenario to be observable three conditions must be met:

- The decay must have occurred recently since the decay products must have traveled less than about $100 \mathrm{Mpc}$ because of the attenuation processes discussed above.

- The mass of this new particle must be well above the observed highest energy (100 EeV range), a hypothesis well satisfied by Grand Unification Theories (GUT) whose scale is around $10^{24}-10^{25} \mathrm{eV}$.

- The ratio of the volume density of this particle to its decay time must be compatible with the observed flux of UHECR.

The $X$-particles may be produced by way of two distinct mechanisms:

- Radiation, interaction or collapse of Topological Defects (TD), producing $X$-particles that decay instantly. In those models the TD are leftovers from the GUT symmetrybreaking phase transition in the very early universe. Quantitative predictions of the TD density that survives a possible inflationary phase rely on a large number of theoretical hypotheses. Therefore they cannot be taken at face value, although the experimental observation of large differences could certainly be interpreted as the signature of new effects.

- Super-massive meta-stable relic particles from some primordial quantum field, pro- 
duced after the now commonly accepted inflationary stage of our Universe. However the ratio of their lifetime to the age of the universe requires a fine tuning with their relative abundance. It is worth noting that in some of those scenarios the relic particles may also act as non-thermal Dark Matter.

In all conceivable Top-Down scenarios, photons and neutrinos dominate at the end of the hadronic cascade. This is the important distinction from the conventional acceleration mechanisms.

\section{THE AUGER DETECTOR}

Large area ground based detectors do not observe the incident cosmic ray directly but the Extensive Air Shower (EAS), a very large cascade of particles, they generate in the atmosphere. All experiments aim to measure, as accurately as possible, the direction of the primary cosmic ray, its energy and its nature. There are two major techniques used. One is to build a ground array of sensors spread over a large area, to sample the EAS particle densities on the ground. The other consists in studying the longitudinal development of the EAS by detecting the fluorescence light emitted by the Nitrogen molecules which are excited by the EAS secondaries.

The Auger Observatories ${ }^{2}$ combine both techniques, with construction starting in the fall of 2000. Once completed in 2006, they will be covering two sites, one in the southern hemisphere (Argentina) and one in the north (Utah, USA). The surface of each site, $3000 \mathrm{~km}^{2}$, will provide statistics of a few tens of events per year above $100 \mathrm{EeV}$. The detector is designed to be fully efficient for showers above $10 \mathrm{EeV}$, with a duty-cycle of $100 \%$. Each station of the ground array is a cylindrical Cerenkov tank of $10 \mathrm{~m}^{2}$ surface and $1.2 \mathrm{~m}$ height filled with filtered water. Because of the size of the array, the stations have to work in a stand-alone mode: they are powered by solar panels and batteries, communication is wireless and timing is provided by the GPS satellites.

The fluorescence telescopes use photo-tubes with a field of view of $1.5^{\circ}$. Each telescope

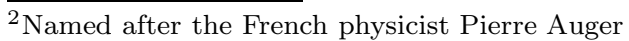

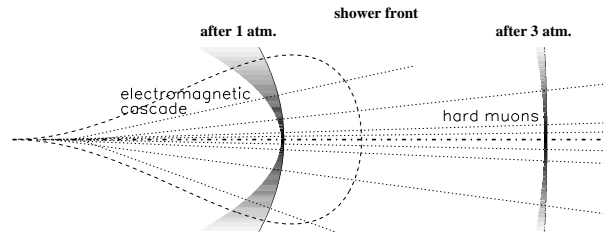

Figure 7. Horizontal shower development [20].

sees an angle of about $30 \times 30$ degrees. On the southern site, three eyes ( 7 telescopes each) will be installed at the periphery of the array and one (12 telescopes) in the middle, in order for the whole array to be visible by at least one of the telescopes. In the hybrid mode $(10 \%$ of the events), the detector is expected to have on average $10 \%$ energy resolution and an angular precision of about $0.3^{\circ}$.

With a total aperture of $14000 \mathrm{~km}^{2} \mathrm{sr}$ (both sites), the Auger Observatory should detect every year of the order of 10000 events above $10 \mathrm{EeV}$ and 100 above $100 \mathrm{EeV}$.

\section{NEUTRINOS}

Although both neutrinos and photons dominate the particle fluxes in Top-Down models, only neutrinos are perfect probes of the characteristics of the sources. High energy neutrinos can also be produced in Bottom Up scenarios as secondaries of hadronic interaction. If AGNs, Radio Galaxy lobes or GRBs are UHECR sources they should produce a substantial flux of neutrinos. Finally, along their path in the universe, hadrons will also produce neutrinos via the pion photo -production processes, the GZK neutrinos.

The first study on the detection of UHE neutrinos with the Auger detector were done by [19,20]. The UHE neutrinos may be detected and distinguished from ordinary hadrons by the shape of the horizontal EAS they produce. At large angles, above $60^{\circ}$, hadronic showers have their electromagnetic part extinguished and only high energy muons survive. Therefore the shower front is very 


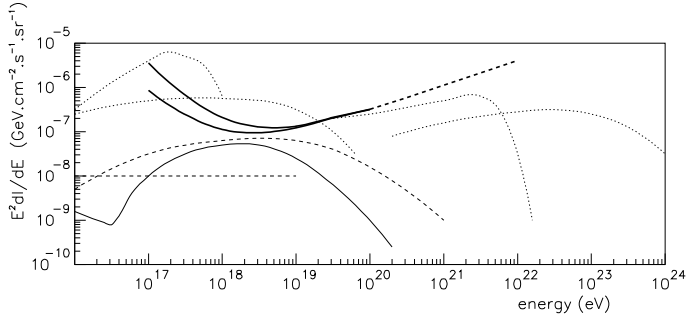

Figure 8. Neutrino fluxes from various sources [18], dotted lines speculative, dashed probable, solid certain. The 2 top thick solid lines represent the Auger sensitivity (0.3 event per year) [20].

flat (radius of curvature is larger than $100 \mathrm{~km}$ ), and very narrow (less than $50 \mathrm{~ns}$ ). Neutrinos interacting deeply in the atmosphere will start a shower above the detector which will appear as a "normal" shower, with a curved front (a few $\mathrm{km}$ ), a large electromagnetic component, and a wider signal (a few microseconds) [see Figure 7]. With such important differences and if the fluxes are high enough, neutrinos will be identified and detected .

Figure 8 shows the expected fluxes from a model calculation by Protheroe 18. The sensitivity limit of the Auger detector defined as 0.3 events per year is also shown. Although each site of the Auger observatory reaches $10 \mathrm{~km}^{3}$ water equivalent of target mass, only the models classified as speculative by the author are expected to yield a detectable signal.

\section{CONCLUSIONS}

The composition, the shape of the energy spectrum, and the distribution of arrivals of UHECR will prove to be powerful tools to distinguish between the different production scenarios.

If UHECR are hadrons accelerated by BottomUp mechanisms, they should point back to their sources, with visible counterparts.

For Top-Down mechanisms and above $100 \mathrm{EeV}$, one should observe a flux of photons and neutrinos as the photon absorption length increases (up to a few $100 \mathrm{Mpc}$ ). Below $100 \mathrm{EeV}$ the spectrum shape and composition will depend on the charac- teristic distance between TD interactions or relic particle decays and Earth, the proton attenuation length and the photon absorption length.

\section{REFERENCES}

1. J.N. Matthews, C.C.H. Jui, Nucl. Phys. Proc. Suppl. B87, (2000) 411.

2. M. Takeda et al., Astrophys.J. 522, (1999) 225; N. Hayashida et al., astro-ph/0008102.

3. K. Greisen, Phys. Rev. Lett. 16 (1966)748.; G.T.Zatsepin, V.A.Kuzmin, JETP Lett. 4 (1966) 78.

4. S. Yoshida, H. Dai, J.Phys. G24 (1998) 905.

5. P. Bhattacharjee, G. Sigl,Phys.Rept. $\mathbf{3 2 7}$ (2000) 109.

6. X. Bertou, M. Boratav, A. Letessier-Selvon, Int.J.Mod.Phys. A15 (2000) 2181.

7. S. Swordy, private communication. Data points from : LEAP, Proton, Akeno, AGASA, Fly's Eye, Haverah Park, Yakutsk.

8. N. Hayashida et al., astro-ph/9906056; see also Y.Uchihori et al., Astropart.Phys. 13 (2000) 151

9. T. Stanev et al., astro-ph/0003484.

10. The Pierre Auger Project Design Report, Fermilab (1995), www.auger.org/admin/.

11. S. Sarkar, astro-ph/0005256.

12. A. Karle et al, Phys. Rev. Lett. B347 (1995) 161.

13. A. M. Hillas, Annual Review Astron. Astrophys. 22 (1984) 425.

14. P. Blasi, R. I. Epstein, A. V. Olinto, astro$\mathrm{ph} / 9912240$.

15. F. Halzen, E. Zas, Ap. J. 488 (1997) 669.

16. P. L. Biermann, Phys. Rev. D51 (1995) 3450.

17. E. Waxman, Nucl. Phys. Proc. Suppl. 87 (2000) 345

18. R.J. Protheroe, astro-ph/9809144

19. G. Parente, E. Zas, astro-ph/9606091; K. S. Capelle, J. W. Cronin, G. Parente, E. Zas, Astropart. Phys. 8 (1998) 321; S. Coutu, X. Bertou, P. Billoir, John Hopkins Workshop (Neutrinos in the Next Millenium), 1999 (sub. to World Scienc.).

20. P. Billoir, 8th International Workshop on Neutrino Telescopes, (1999) 111; 